\section{ORIGINAL RESEARCH}

\author{
G.A. Christoforidis \\ C. Karakasis \\ Y. Mohammad \\ L.P. Caragine \\ M. Yang \\ A.P. Slivka
}

\title{
Predictors of Hemorrhage Following Intra-Arterial Thrombolysis for Acute Ischemic Stroke: The Role of Pial Collateral Formation
}

BACKGROUND AND PURPOSE: The extent of pial collateral formation during acute ischemic stroke has been shown to influence outcomes. This study examines whether angiographic assessment of pial collateral formation is predictive of hemorrhagic transformation following intra-arterial thrombolysis (IAT) for acute ischemic stroke.

MATERIALS AND METHODS: Rates of any hemorrhage and significant hemorrhage ( $>25 \mathrm{~mL}$ ) were reviewed in 104 consecutive patients who underwent IAT following acute ischemic stroke. The influence of the anatomic extent of pial collateral formation on the rates of hemorrhage and significant hemorrhage relative to known predictors for hemorrhagic transformation (presenting systolic blood pressure, blood glucose level, platelet level, and National Institutes of Health Stroke Scale [NIHSS] score, history of diabetes, time to treatment, age, sex, occlusion site, and extent of reperfusion) was analyzed by using logistic regression models.

RESULTS: Rates of any hemorrhage and significant hemorrhage were 25.2\% (26/104) and 9.7\% (10/104), respectively. The rate of significant hemorrhage was $25.0 \%(8 / 32)$ in patients with poor pial collaterals and $2.78 \%(2 / 72)$ in those with good pial collaterals ( $P=.0004$, Pearson correlation). The rate of any hemorrhage was also significantly higher in patients with poor pial collaterals $(40.6 \%$ versus $18.1 \% ; P=.0142$, Pearson correlation). Logistic regression analyses revealed that pial collateral formation (odds ratio $[O R]=3.04$ ), history of diabetes $(\mathrm{OR}=4.83$ ), platelets $<200,000 / \mu \mathrm{L}(\mathrm{OR}=$ $2.95)$, and time to treatment $<3$ hours $(O R=12.0)$ were statistically significant predictors of hemorrhage, whereas pial collateral formation $(O R=13.1)$ and platelets $<200,000 / \mu \mathrm{L}(\mathrm{OR}=8.1$ ) were statistically significant predictors of significant hemorrhage.

CONCLUSIONS: Poor pial collateral formation is associated with higher incidence and larger size of hemorrhage following IAT.

ntra-arterial thrombolysis (IAT) has the potential to rescue ischemic brain tissue within a penumbra zone surrounding irreversibly infarcted brain. ${ }^{1,2}$ Intracerebral hemorrhage $(\mathrm{ICH})$ is considered the most devastating complication of thrombolytic treatment for acute ischemic stroke. A variety of imaging methods has been used to assess the ischemic penumbra and the infarcted tissue on presentation to determine which patients are more likely to benefit from thrombolytic treatment. Furthermore, these methods have been shown potentially to predict hemorrhagic transformation (HT) following thrombolytic treatment. ${ }^{3-8}$ As such, imaging studies that assess salvageable brain and brain that can no longer be salvaged also have the potential to weigh the risk-benefit ratio of thrombolytic treatment. Studies such as diffusion/perfusion MR imaging are not always available in a timely fashion. Angiographic assessment of pial collateral formation can be used to estimate whether a significant amount of tissue may be rescued $^{9}$ and is always available at the time of IAT without any delay in the delivery of treatment. This study sought to determine whether patients with good pial collateral formation

Received May 7, 2008; accepted after revision July 10

From the Departments of Radiology (G.A.C., C.K., M.Y.), Neurology (Y.M., A.P.S.), and Neurosurgery (L.P.C.), The Ohio State University College of Medicine, Columbus, Ohio.

Paper previously presented in part at: International Stroke Conference, February 16-18, 2006, Kissimmee, Fla; and Annual Meeting of the American Society of Neuroradiology, May 6-12, 2006, San Diego, Calif

Please address correspondence to Gregory A. Christoforidis, MD, 627 Means Hall, 1654 Upham Dr, The Ohio State University Medical Center, Columbus, $\mathrm{OH}$ 43210; e-mail: greg.christoforidis@osumc.edu

DOI 10.3174/ajnr.A1276 during acute ischemic stroke documented on angiography have lower hemorrhage rates than patients with poor pial collateral formation in relation to other predictors for HT.

\section{Materials and Methods}

This study included consecutive patients who underwent IAT by using recombinant tissue plasminogen activator (tPA) ( $\leq 100 \mathrm{mg})$, urokinase (UK) ( $\leq 1$ million units), and pro-urokinase (pro-UK) ( $\leq 9 \mathrm{mg}$ ) from May 1995 until April 2007. Data were collected by using an institutional review board-approved protocol. Patients seen within 6 hours of symptoms who were considered thrombolytic candidates by using clinical, laboratory, CT, and angiographic criteria derived from the Prolyse in Acute Cerebral Thromboembolism (PROACT) trial underwent screening cerebral angiography. ${ }^{2}$ Unlike the PROACT study, this study also included patients with ischemic stroke involving territories other than the middle cerebral artery (MCA) and patients older than 85 years of age. Patients with basilar artery (BA) occlusion were included in this study if they were treated within 12 hours of symptom onset, if initial symptoms were mild (nausea or National Institutes of Health Stroke Scale [NIHSS] score, $<4$ ), and if their condition deteriorated (NIHSS score $>4$ ) within 6 hours of treatment onset. The presenting NIHSS score was the last one recorded before treatment. Some patients who met inclusion and exclusion criteria before angiography did not receive treatment either because the angiographic findings were negative or their symptoms resolved in the interim between clinical assessment and angiography.

Patients who underwent embolectomy were excluded. Patients underwent angiography and thrombolytic treatment with local anesthesia. The microcatheter was embedded within the occluding throm- 
bus; however, microcatheter manipulation was at the discretion of the operator. Most patients receiving tPA were not anticoagulated following treatment, whereas patients receiving UK or pro-UK were. Treatment end points were considered to be the following: 1) angiographic signs of hemorrhage; 2) complete reperfusion with complete recanalization; 3 ) if infusion was begun before 6 hours but the patient did not experience recanalization by 6 hours, continued infusion was left to the discretion of the physicians involved. For the purposes of this study, CT scans and angiographic information needed to be available.

All patients underwent CT 24-48 hours following symptom onset. In addition, patients who developed a hemorrhage or new symptoms underwent additional CT while in the hospital. Acute infarction on 24- to 48-hour CT was defined as a new hypoattenuated region relative to the pretreatment CT. HT was defined as a new hyperattenuated region identified on any follow-up CT scan before patient discharge. Patients who developed a new hyperattenuated region that completely cleared within 48 hours were considered to have contrast extravasation and were not considered to have HT. Foci of hemorrhage were traced out on axial cross-sectional images by a Certificate of Added Qualification-certified neuroradiologist, and areas were calculated by using Impax image analysis software (Agfa, Ridgefield Park, NJ).

Hemorrhage volumes were then determined by multiplying crosssectional areas by section thickness and adding them up as previously described. ${ }^{9,10}$ Significant hemorrhage was defined as any HT with a calculated volume of $\geq 25 \mathrm{~mL}$. Significant hemorrhage has been shown to be objectively associated with clinical deterioration from ICH following IAT when compared with other markers of clinically significant hemorrhage such as symptomatic hemorrhage or parenchymal hematoma involving $>30 \%$ of the infarcted territory. ${ }^{10}$ Symptomatic hemorrhage was defined as any hemorrhage associated with an increase in NIHSS score of $\geq 4$ between the time of presentation and 24-36 hours following ictus. ${ }^{8}$

Angiograms of all patients were reviewed for occlusion site, pial collateral formation, and reperfusion by an interventional neuroradiologist who was blinded to all clinical information during this review. Pial collaterals were graded on a 5-point scale based on anatomic extent (Fig 1) as defined elsewhere, with good pial collaterals being grades 1 and 2 and poor being grades $3-5 .{ }^{9}$ A score of 1 was assigned if collaterals reconstituted the distal portion of the occluded vessel segment (ie, if there was M1 segment occlusion, the M1 segment distal to the occlusion was reconstituted). A score of 2 was assigned if collaterals reconstituted vessels in the proximal portion of the segment adjacent to the occluded vessel (ie, if there was M1 segment occlusion with reconstitution to the proximal M2 vessel segments). A score of 3 was assigned if collaterals reconstituted vessels in the distal portion of the segment adjacent to the occluded vessel (ie, if there was M1 segment occlusion with reconstitution to the distal portion of the M2 vessel segments). A score of 4 was assigned if collaterals reconstituted vessels 2 segments distal to the occluded vessel (ie, if there was M1 segment occlusion with reconstitution up to the M3 segment branches). A score of 5 was assigned if there was little or no significant reconstitution of the territory of the occluded vessel. Occlusions at the internal carotid bifurcation with a patent anterior communicating artery (AcomA) were scored similar to M1 occlusions because the anterior cerebral artery (ACA) A2 segment was antegradely reconstituted via the AcomA.

Intraobserver agreement for this scoring method for pial collateral formation was identical in $87 \%$ of patients and differed by 1 scoring point in the rest of the patients $(\kappa=0.81, \mathrm{SE}=0.065)$. In the case of

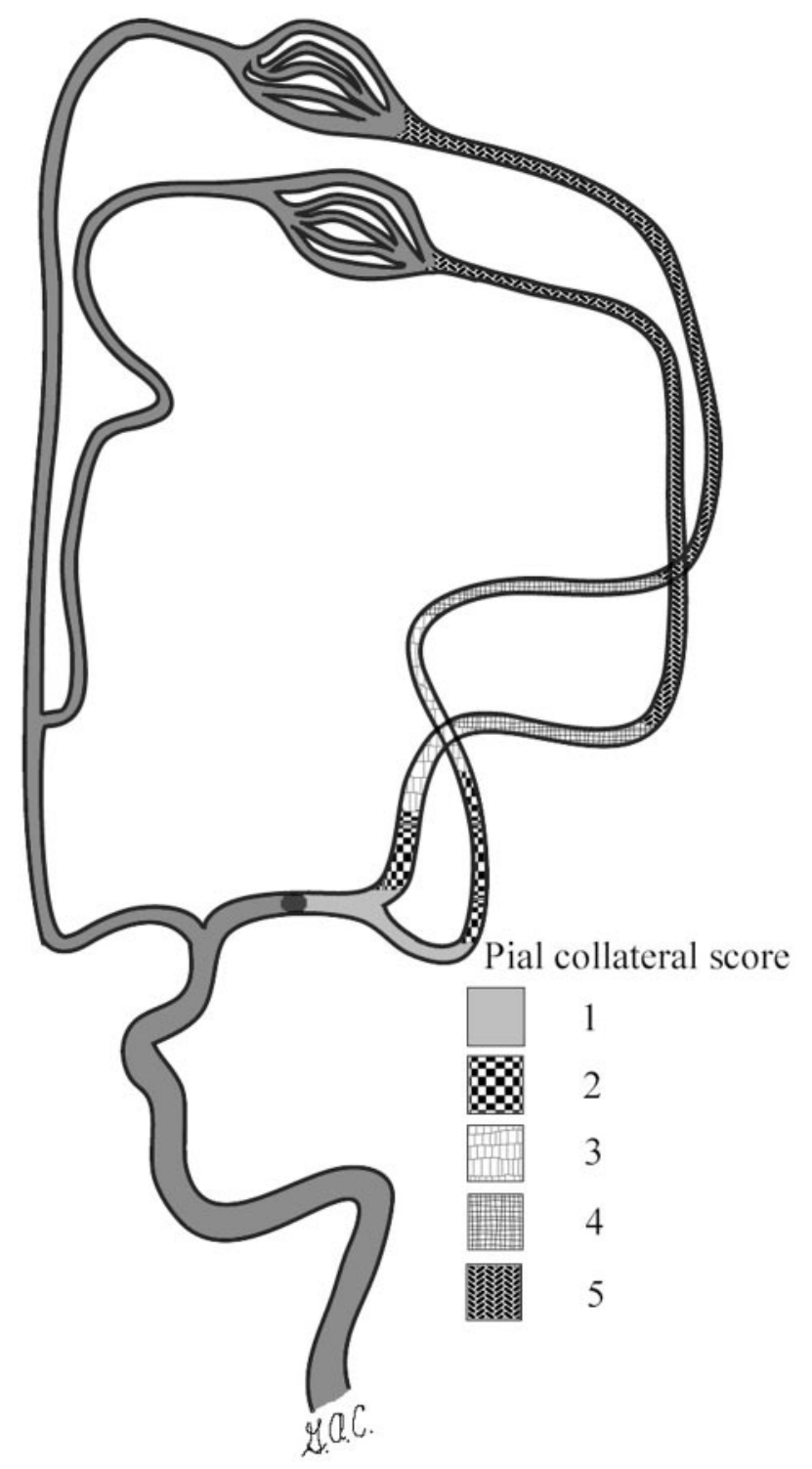

Fig 1. This diagram depicts the scoring of the anatomic extent of pial collateral formation from the ACA territory to the MCA territory during occlusion of the M1 segment. Scoring corresponds to angiographically visible retrograde opacification of the MCA segments on the delayed venous phase. ${ }^{9}$ Each distinctly textured segment depicts the furthest extent of retrograde opacification identified on cerebral angiography for each pial collateral score.

MCA occlusion, pial collateral formation from the ACA was always evaluated. Posterior cerebral artery collaterals were not consistently evaluated. All potential sources from the circle of Willis and pial collateral formation were evaluated for internal carotid artery (ICA) and BA occlusions. Good pial collaterals in this study were equivalent to grades 3 and 4 described by Higashida and Furlan. ${ }^{11}$ Occlusions were considered proximal if the site of occlusion was at the ICA, BA, or M1, A2, or P1 segments. Occlusions were considered distal if the occlusion was at or more distal to the M2, A3, or P2 cerebral arterial segments or involved cerebellar branches.

Reperfusion was assessed as a percentage of the affected vascular territory that was revascularized by taking the ratio of the number of reperfused branches of the affected territory to the number of branches occluded before recanalization. In this study, complete reperfusion was considered to be complete recanalization with complete reperfusion. ${ }^{12}$ Arteriograms were reviewed to assess reperfusion 
on the basis of the modified thrombolysis in myocardial ischemia score (Mori score). ${ }^{13}$ Patients with no reperfusion were classified as Mori 0 , patients between $0 \%$ and $50 \%$ reperfusion were classified as Mori 1, patients between $50 \%$ and $100 \%$ reperfusion were classified as Mori 2, and patients with 100\% reperfusion were classified as Mori 3.

\section{Statistical Analysis}

Predictors for significant hemorrhage, symptomatic hemorrhage, and any hemorrhage were identified by using a multivariate logistic regression model. Predictive variables entered into the model included the following: pial collateral formation, presenting systolic blood pressure, presenting serum glucose level, history of diabetes, platelet level, presenting NIHSS score, time to treatment, age, sex, occlusion site, and extent of reperfusion. All these factors have been previously shown to be associated with either cerebral hemorrhage following thrombolytic treatment or outcome. ${ }^{2,5,6,9,14-22}$ All continuous variables were tested for normal distribution by using the Shapiro-Wilk test for normality. Nonparametric variables were dichotomized for logistic regression analysis. Dichotomization was derived from previous studies. ${ }^{2,5,9,14-22}$ All risk factors with $P<.05$ were entered into the final model as predictors of hemorrhage volume and hemorrhage rate by using backward selection. Rates of hemorrhage and median values for statistically significant variables identified on both regression analyses were then compared. Statistical significance was determined by using the Wilcoxon rank sum test for nonparametric data and the Pearson correlation for contingency analysis.

\section{Results}

A total of 104 patients (56 men, 48 women) of 112 who fulfilled inclusion criteria and received IAT therapy at our institution between May 1995 and April 2007 were reviewed. In 8 patients, pial collateral formation could not be assessed because angiographic acquisitions did not include the venous phase. Mean age was 68.0 years (range, 33-90 years), median presentation NIHSS score was 16 (range, 4-30), and median time to treatment was 285 minutes (range, 46-720 minutes). Twenty of the 104 patients had a history of diabetes. Three patients with BA occlusion exceeded 360 minutes under the criteria described previously. tPA was administered to 81 (78\%); UK, to $21(20 \%)$; and pro-UK, to $2(2 \%)$ patients. Occlusion sites included the following: MCA $(n=78)$, ICA $(n=14)$, BA $(n=8)$, ACA $(n=3)$, and posterior inferior cerebellar artery $(n=1)$. Of these occlusion sites, 80 were proximal and 24 were distal. Median presenting platelets were $216,000 / \mu \mathrm{L}(104,000-501,000 / \mu \mathrm{L})$; median presenting glucose level was $119 \mathrm{mg} / \mathrm{dL}$ (range, 83-393 mg/dL), and mean presenting systolic blood pressure was $146.3 \mathrm{~mm} \mathrm{Hg}(\sigma=$ $25.8)$. Twenty-six patients (25\%) developed a hemorrhage of which 10 were $>25 \mathrm{~mL}$ in volume and 7 were associated with an increase in NIHSS score of $\geq 4$. There were a total of 9 patients with posterior circulation infarctions. Five of these patients exceeded the 6-hour window (please see above for clinical criteria for entry for such patients). None had a symptomatic hemorrhage or a hemorrhage $>25 \mathrm{~mL}$. One of these patients had a small hemorrhage $(7.3 \mathrm{~mL})$, which was not associated with clinical deterioration.

Figure 2 demonstrates that the rates of any hemorrhage and any significant hemorrhage increase as pial collateral grades worsen. Logistic regression analyses displayed in Tables 1 and 2 indicate that pial collateral scores, platelet levels, and a his-

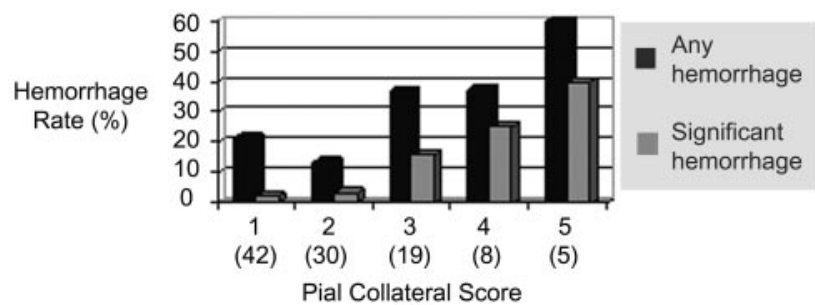

(n)

Fig 2. Bar graph displays the rates of any hemorrhage (black) and significant hemorrhage (hemorrhage volume $>25 \mathrm{~mL}$, gray) based on the pial collateral score.

\begin{tabular}{lccc}
\hline \multicolumn{4}{l}{ Table 1: Logistic regression analysis for any hemorrhage } \\
\hline Predictor & $\mathrm{OR}(95 \% \mathrm{CI})$ & $\begin{array}{c}\text { Estimate } \\
\text { (SE) }\end{array}$ & $P>\chi^{2 *}$ \\
\hline Poor pial collateral score & $3.03(1.09-8.71)$ & $1.11(0.52)$ & .0342 \\
Platelets <200K† & $2.95(1.06-8.57)$ & $1.08(0.53)$ & .0403 \\
Diabetes & $4.82(1.49-16.9)$ & $0.787(0.306)$ & .0100 \\
Time to treat $>180$ min & $12.0(1.77-253)$ & $2.47(1.17)$ & .0333 \\
Intercept & & $-0.81(0.52)$ & .1138 \\
\hline
\end{tabular}

Note:-OR indicates odds ratio.

* Variables tested with $P>.05$ were rejected using backward selection (whole-model test, $\left.P<.0001 ; r^{2}=0.202\right)$.

$+200 \mathrm{~K}=200,000 / \mu \mathrm{L}$.

Table 2: Logistic regression analysis for significant hemorrhage (volume $>25 \mathrm{~mL}$ )

\begin{tabular}{lccc}
\hline Predictive factor & OR $(95 \% \mathrm{CI})$ & $\begin{array}{c}\text { Estimate } \\
\text { (SE) }\end{array}$ & $P>\chi^{2 *}$ \\
\hline Poor pial collateral score & $13.1(2.84-96.0)$ & $2.57(0.860)$ & .0028 \\
Platelets <200K & $8.14(1.73-60.3)$ & $2.10(0.868)$ & .0157 \\
Intercept & & $0.190(0.520)$ & .715 \\
\hline
\end{tabular}

Note:- $0 \mathrm{R}$ indicates odds ratio; $\mathrm{Cl}$, confidence interval.

* Variables tested with $P>.05$ were rejected using backward selection (whole-model test, $\left.P<.0001 ; r^{2}=0.202\right)$.

\begin{tabular}{|c|c|c|c|c|}
\hline & & $P$ & & $P$ \\
\hline Parameter & $\begin{array}{l}\text { Rate of Any } \\
\text { Hemorrhage }\end{array}$ & $\begin{array}{c}\text { Value } \\
\text { (Pearson) }\end{array}$ & $\begin{array}{c}\text { Rate of Significant } \\
\text { Hemorrhage }\end{array}$ & $\begin{array}{c}\text { Value } \\
\text { (Pearson) }\end{array}$ \\
\hline$\overline{\text { Total }}$ & $26 / 104(25.0 \%)$ & & $10 / 104(9.61 \%)$ & \\
\hline Good collaterals & $13 / 72(18.1 \%)$ & .0142 & $2 / 72(2.78 \%)$ & .0004 \\
\hline Poor collaterals & $13 / 32(40.6 \%)$ & & $8 / 32(25.0 \%)$ & \\
\hline Platelets $<200 \mathrm{~K}$ & $16 / 42(38.1 \%)$ & .0111 & $8 / 42(19.0 \%)$ & .0072 \\
\hline Platelets $>200 \mathrm{~K}$ & $10 / 62(16.1 \%)$ & & $2 / 62(3.23 \%)$ & \\
\hline
\end{tabular}

* Shows significantly higher rates of any hemorrhage and significant hemorrhage (volume $>25 \mathrm{~mL}$ ) among patients with poor pial collaterals and platelet levels $<200,000 / \mu \mathrm{L}$.

tory of diabetes were significant contributors to hemorrhage in this study. Only poor pial collateral formation and platelet counts $<200,000 / \mu \mathrm{L}$ were statistically significant predictors of significant hemorrhage in this study. Table 3 demonstrates the effects of pial collateral score and platelet level on the rate of any hemorrhage and the rate of significant hemorrhage. Patients with significant hemorrhage had median platelet counts of $178,000 / \mu \mathrm{L}$ (range, $154,000-250,000 / \mu \mathrm{L}$ ), whereas those without significant hemorrhage had a median platelet count of $227,000 / \mu \mathrm{L}$ (range, 104,000-501,000/ $\mu \mathrm{L}$ ). This difference was statistically significant $(P=.0129,2$-sample Wilcoxon rank sum test). Seven patients in this series of 104 patients were 

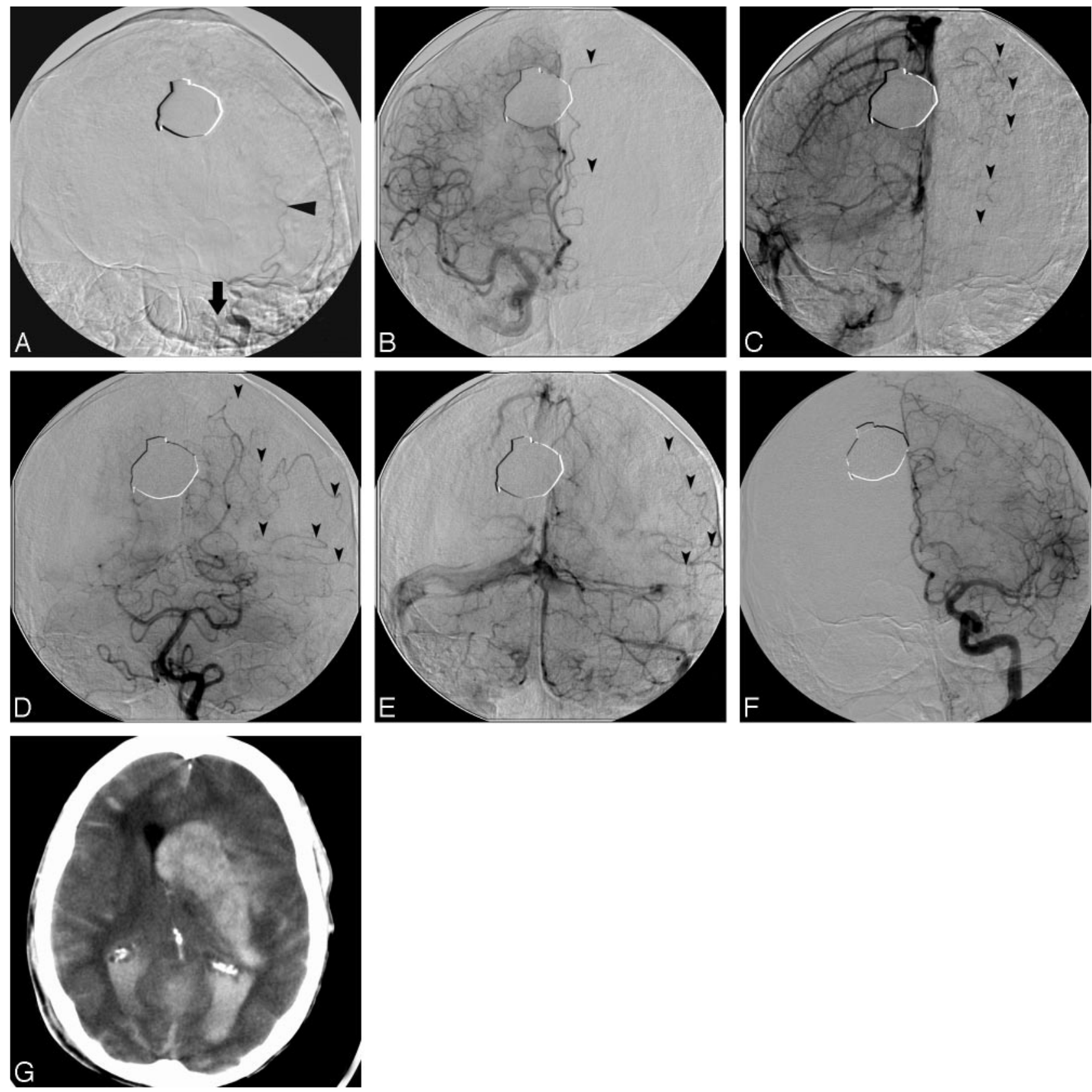

Fig 3. Large hemorrhagic conversion following reperfusion in a patient with distal left internal carotid occlusion with poor pial collateral formation. $A$, Left internal carotid arteriogram

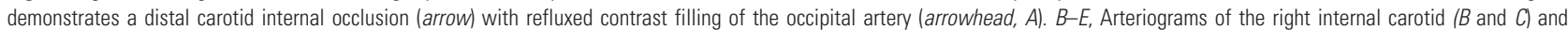
left vertebral arteries $(D$ and $E$ ) demonstrate no circle of Willis collaterals on either the late arterial phase (arrowheads, $B$ and $D$ ) or the late venous phase (arrowheads, $C$ and $E$ ). Poor pial collateral formation reconstitutes, at best, the M4 branches of the MCA (arrowheads, $D$ and $E$ ). $F$ and $G$, Twenty-four hours following reperfusion ( $F$, the patient experienced a large hemorrhage $(G)$ and subsequently died.

found to have symptomatic hemorrhage. Logistic regression analysis for symptomatic hemorrhage by using all predictive variables identified only poor pial collateral formation as a statistically significant $(P=.0286)$ predictor for symptomatic hemorrhage with an odds ratio of 6.8 (95\% confidence intervals, $1.4-49.7$ )

\section{Discussion}

HT is assumed to occur following reperfusion into ischemically injured vessels, which then allow varying degrees of blood extravasation through the damaged blood-brain barrier. ${ }^{23-25}$ Clinical factors shown to be associated with a higher incidence of HT include profound clinical deficit as represented by a high presenting NIHSS score, longer time to treatment, use of anticoagulants, lower platelet levels, history of diabetes, elevated systolic blood pressure, and elevated presenting glucose levels. ${ }^{2,5,9,14-22}$ Imaging findings predictive of HT have included larger territory at risk for infarction as indicated by either a large diffusion abnormality on MR imaging, ${ }^{4,6}$ a larger perfusion defect (MR imaging, CT, single-photon emission CT), ${ }^{3,6}$ early CT hypoattenuation, ${ }^{5,7,8,26}$ or a large infarct volume. $^{10}$

Pial collateral flow to an ischemic territory may improve clinical outcomes by sustaining ischemic tissue for longer pe- 

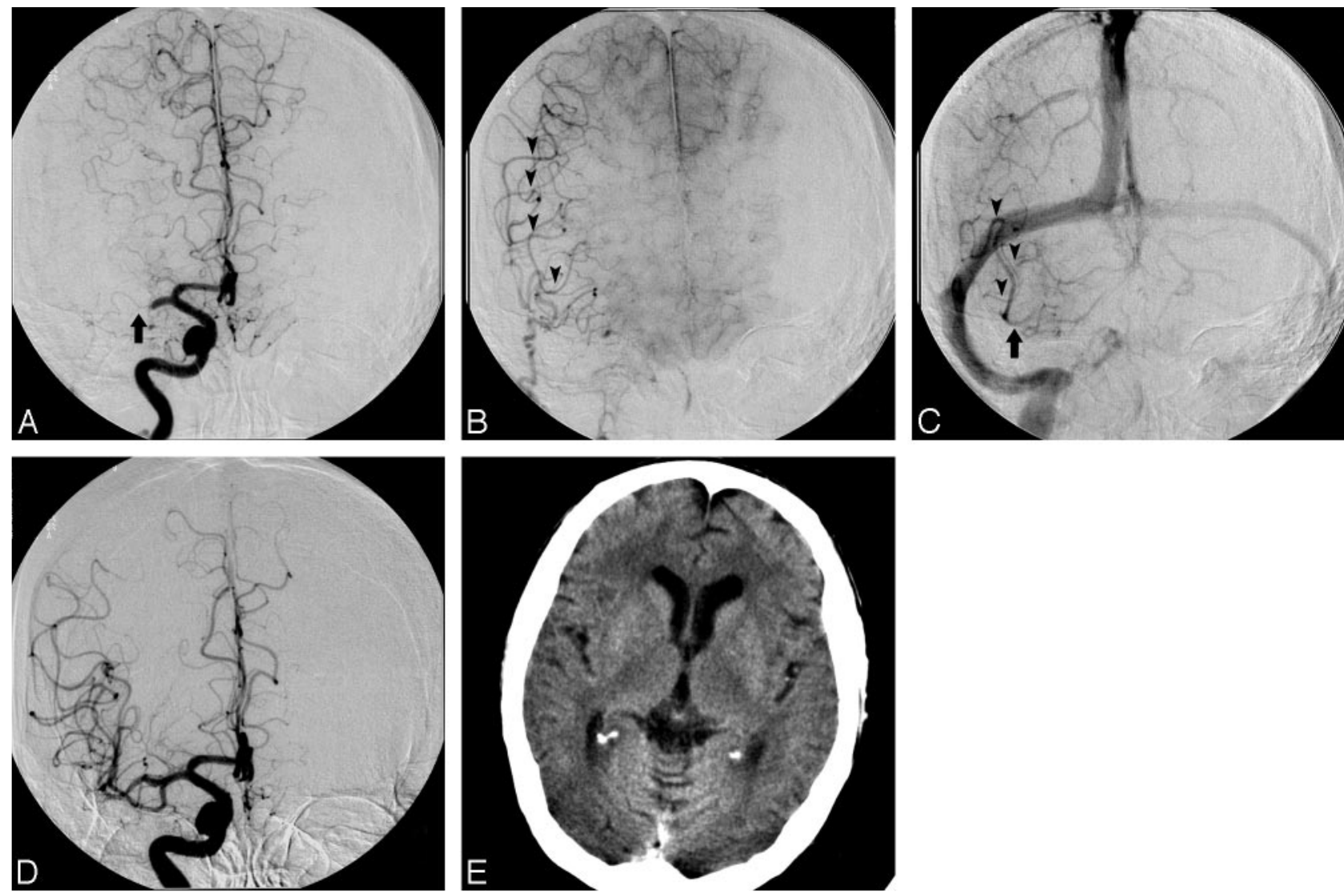

Fig 4. Arterial $(A)$, parenchymal $(B)$, and late venous $(C)$ phase arteriograms in a patient with right MCA occlusion $(A$, arrow) demonstrate good pial collateral formation $(B$ and $C$, arrowheads with reconstitution of the entire MCA territory, including the M1 segment (arrow, $C$ ). $D$ and $E$, Following reperfusion $(D)$, the patient had no hemorrhage or infarction $(E)$.

riods of time, thus extending the time within which the ischemic tissue may be rescued via recanalization. Poor pial collateral formation is associated with larger infarct volumes and worse clinical outcomes. ${ }^{9}$ Furthermore, it is generally agreed that the penumbra zone identified on perfusion studies is sustained by pial collateral blood flow and that poor pial collateral formation is associated with a larger diffusion abnormality. ${ }^{6}$ Because pial collateral formation can act as an indirect measure of perfusion to a territory, it can follow that poor pial collateral formation should be associated with a higher risk of HT. However, larger infarct volumes and delayed perfusion to a territory have been shown to increase the risk for symptomatic hemorrhage or hemorrhage rate. ${ }^{10,26}$ A literature search by using MEDLINE and PubMed indicated that no studies have been published showing that poor pial collateral formation can act as an independent predictor of hemorrhage rate following IAT. Data presented here indicate that poor pial collateral formation identified on angiography independently correlates with increased rates of any hemorrhage as well as hemorrhage volumes of $\geq 25 \mathrm{~mL}$ (Tables 1-3, Figs 2-4), following IAT.

In this study, clinical factors found to be predictive of HT included poor pial collateral formation, a history of diabetes, time to treatment $>3$ hours, and platelets below $200,000 / \mu \mathrm{L}$. Baseline hyperglycemia has been implicated in previous studies as an independent risk factor for HT; this occurs presumably by the effect of high glucose on the microcirculation, which enhances the effect of ischemic injury and damage to the blood-brain barrier. ${ }^{27-29}$ Although baseline hyperglycemia was included as a potential predictor, it is suggested that there was statistical interaction with a history of diabetes that resulted in its exclusion from the final model. A platelet level below a threshold of $100,000 / \mu \mathrm{L}$ has traditionally served as a contraindication for thrombolysis. ${ }^{2}$ In this study, platelet levels below $200,000 / \mu \mathrm{L}$ were found to be independently associated with higher hemorrhage rates and rates of significant hemorrhage (Tables 1-3). On the basis of this finding, one can hypothesize that administration of platelets immediately following thrombolytic treatment may act to prevent hemorrhagic complications.

Proximal occlusions involving end perforators are also expected to result in a larger hemorrhage rate. In this study, they were not found to be significantly associated with HT, possibly because of the sample size. Hemorrhage rates in 17 patients whose treatment started within 3 hours of symptom onset were much lower than those whose treatment started $>3$ hours following symptom onset. In our experience, patients with poor pial collateral formation have a tendency to present with higher NIHSS scores. Interaction between variables may, in part, help explain why the NIHSS score was not found to be a statistically significant predictor of hemorrhagic transformation on logistic regression analysis in this study. A larger sample size would help gain more insight.

Time may act as a predisposing factor for hemorrhage following thrombolytic delivery in the setting of acute ischemic stroke. The fact the few patients who were treated beyond the 6-hour time window did not develop a significant or a symptomatic hemorrhage suggests that under certain circum- 
stances, thombolytics may be beneficial beyond the 6-hour time window. In this study, only patients with BA occlusion who presented with minor symptoms (NIHSS score, $<4$ ) and whose condition deteriorated significantly within 6 hours of treatment onset were included.

This study has several limitations that deserve mention. Evaluation of the extent of pial collateral formation and hemorrhage volumes was derived retrospectively, which could lead to misclassification and selection bias. This was controlled by using a consecutive series, blinding the investigators, and using pre-established definitions for pial collaterals and hemorrhage volumes to categorize the patients. A larger patient population is needed to provide a more robust analysis of hemorrhagic transformation in the setting of IAT. This study did not exclude patients on the basis of the location of infarction. Although this choice may introduce bias, it is consistent with the patient population mix in multicenter trials evaluating outcomes for intravenous tPA. ${ }^{8,15,18}$ There was no indication from these studies that hemorrhage rates differed between patients with anterior-versus-posterior circulation infarction. Finally more than 1 type of thrombolytic agent was used in this study (21 patients received UK, 2 patients received pro-UK, and 81 patients received tPA). There is no indication, however, that hemorrhage rates were significantly different between the various agents.

\section{Conclusions}

Poor pial collateral formation identified on angiography is associated with a higher incidence and larger size of hemorrhage following IAT. In view of the fact that these patients also tend to have poor clinical outcomes even when no hemorrhage is present, curtailing aggressive thrombolytic treatment may be warranted. As such, patients with pial collateral grades 4 or 5 may be considered poor candidates for IAT treatment. Although nonthrombolytic means (ie, mechanical thrombolysis) for reperfusion may serve as alternative methods for reperfusion, it must be cautioned that these patients may still be at high risk for hemorrhagic transformation. Finally, patients with platelet levels below $200,000 / \mu \mathrm{L}$ had a higher predisposition toward significant hemorrhage following thrombolytic therapy. Whether exogenous platelet administration could be used to attenuate this effect following thrombolytic treatment remains to be investigated.

\section{Acknowledgments}

We acknowledge Peggy Notestine, BS, and Hoda Jradi, MS, for their help.

\section{References}

1. Lisboa RC, Jovanovic BD, Alberts MJ. Analysis of the safety and efficacy of intra-arterial thrombolytic therapy in ischemic stroke. Stroke 2002;33:2866-71

2. Furlan A, Higashida R, Wechler L, et al. Intra-arterial prourokinase for acute ischemic stroke: The PROACT II study—a randomized controlled trial. Prolyse in Acute Cerebral Thromboembolism. JAMA 1999;282:2003-11

3. Tong DC, Adami A, Moseley MP, et al. Prediction of hemorrhagic transformation following acute stroke: role of diffusion- and perfusion-weighted magnetic resonance imaging. Arch Neurol 2001;58:587-93
4. Selim M, Fink JN, Kumar S, et al. Predictors of hemorrhagic transformation after intravenous recombinant tissue plasminogen activator: prognostic value of the initial apparent diffusion coefficient and diffusion-weighted lesion volume. Stroke 2002;33:2047-52

5. Lansberg MG, Albers GW, Wijman CA. Symptomatic intracerebral hemorrhage following thrombolytic therapy for acute ischemic stroke: a review of the risk factors. Cerebrovasc Dis 2007;24:1-10. Epub 2007 May 22.

6. Liebeskind DS. Collaterals in acute stroke: beyond the clot. Neuroimaging Clin N Am 2005;15:553-73

7. Tanne D, Kasner SE, Demchuk AM, et al. Markers of increased risk of intracerebral hemorrhage after intravenous recombinant tissue plasminogen activator therapy for acute ischemic stroke in clinical practice: the Multicenter rt-PA Stroke Survey. Circulation 2002;105:1679-85

8. Intracerebral hemorrhage after intravenous $t-P A$ therapy for ischemic stroke: The NINDS t-PA Stroke Study Group. Stroke 1997;28:2109-18

9. Christoforidis GA, Mohammad Y, Kehagias D, et al. Angiographic assessment of pial collaterals as a prognostic indicator following intra-arterial thrombolysis for acute ischemic stroke. AJNR Am J Neuroradiol 2005;26:1789-97

10. Christoforidis GA, Slivka A, Mohammad Y, et al. Size matters: hemorrhage volume as an objective measure to define significant intracranial hemorrhage associated with thrombolysis. Stroke 2007;38:1799-804

11. Higashida RT, Furlan AJ. Trial design and reporting standards for intra-arterial cerebral thrombolysis for acute ischemic stroke. Stroke 2003;34:109-37

12. Khatri P, Neff J, Broderick JP, et al, for the IMS-I Investigators. Revascularization end points in stroke interventional trials: recanalization versus reperfusion in IMS-I. Stroke 2005;35:2400-03

13. Arnold M, Nedeltchev K, Remonda L, et al. Recanalisation of middle cerebra artery occlusion after intra-arterial thrombolysis: different recanalisation grading systems and clinical functional outcome. J Neurol Neurosurg Psychiatry 2005;76:1373-76

14. Hacke W, Kaste M, Fieschi C, et al. Randomised double-blind placebo-controlled trial of thrombolytic therapy with intravenous alteplase in acute ischaemic stroke (ECASS II): Second European-Australasian Acute Stroke Study Investigators. Lancet 1998;353:1245-51

15. Clark WM, Wissman S, Albers GW, et al. Recombinant tissue-type plasminogen activator (alteplase) for ischemic stroke 3 to 5 hours after symptom onset: The ATLANTIS Study - a randomized controlled trial. Alteplase Thrombolysis for Acute Noninterventional Therapy in Ischemic Stroke. JAMA 1999;282:2019-26

16. Kidwell CS, Saver JL, Carnado J, et al. Predictors of hemorrhagic transformation in patients receiving intra-arterial thrombolysis. Stroke 2002;33:717

17. Del Zoppo G, Poeck K, Pessin M, et al. Recombinant tissue plasminogen activator in acute thrombotic and embolic stroke. Ann Neurol 1992;32:78-86

18. Larrue V, von Kummer R, del Zoppo G, et al. Hemorrhagic transformation in acute ischemic stroke: potential contributing factors in the European Cooperative Acute Stroke Study. Stroke 1997;28:957-60

19. Levy D, Brott T, Haley C, et al. Factors related to intracranial hematoma formation in patients receiving tissue-type plasminogen activator for acute ischemic stroke. Stroke 1994;25:291-97

20. Suarez J, Sunshine J, Tarr R, et al. Predictors of clinical improvement, angiographic recanalization, and intracranial hemorrhage after intra-arterial thrombolysis for acute ischemic stroke. Stroke 1999;30:2094-100

21. Demchuk A, Morgenstern L, Krieger D, et al. Serum glucose level and diabetes predict tissue plasminogen activator-related intracerebral hemorrhage in acute ischemic stroke. Stroke 1999;30:34-39

22. Kase CS, Furlan AJ, Wechsler LR, et al. Cerebral hemorrhage after intra-arterial thrombolysis for ischemic stroke: the PROACT II trial. Neurology 2001;57:1603-10

23. Gasche Y, Copin JC, Sugawara T, et al. Matrix metalloproteinase inhibition prevents oxidative stress-associated blood-brain-barrier disruption after transient focal cerebral ischemia. J Cereb Blood Flow Metab 2001;21:1393-400

24. Castellanos M, Leira R, Serena J, et al. Plasma metalloproteinase-9 concentration predicts hemorrhagic transformation in acute ischemic stroke. Stroke 2003;34:40-46

25. Khatri $\mathrm{P}$, Wechsler LR, Broderick JP. Intracranial hemorrhage associated with revascularization therapies. Stroke 2007;38:431-40

26. Hacke W, Kaste M, Fieschi C, et al. Intravenous thrombolysis with recombinant tissue plasminogen activator for acute hemispheric stroke: The European Cooperative Acute Stroke Study (ECASS). JAMA 1995;274:1017-25

27. Dietrich WD, Alonso O, Busto R. Moderate hyperglycemia worsens acute blood-brain-barrier injury after forebrain ischemia in rats. Stroke 1993;24:111-16

28. Kawai N, Keep RF, Betz AL. Hyperglycemia and the vascular effects of cerebral ischemia. Stroke 1997;28:149-54

29. Hamann GF, del Zoppo GJ, von Kummer R. Hemorrhagic transformation of cerebral infarction: possible mechanisms. Thromb Haemost 1999;82:92-94 